\title{
Fractal Dynamical Model of Vehicular Traffic Flow within the Local Fractional Conservation Laws
}

\author{
Long-Fei Wang, ${ }^{1}$ Xiao-Jun Yang, ${ }^{2}$ Dumitru Baleanu, ${ }^{3,4,5}$ Carlo Cattani, $^{6}$ and Yang Zhao ${ }^{7}$ \\ ${ }^{1}$ School of Highway, Chang'an University, Xian 710064, China \\ ${ }^{2}$ Department of Mathematics and Mechanics, China University of Mining and Technology, Xuzhou Campus, \\ Xuzhou, Jiangsu 221008, China \\ ${ }^{3}$ Department of Chemical and Materials Engineering, Faculty of Engineering, King Abdulaziz University, \\ P.O. Box 80204, Jeddah 21589, Saudi Arabia \\ ${ }^{4}$ Department of Mathematics and Computer Sciences, Faculty of Arts and Sciences, Cankaya University, \\ 06530 Ankara, Turkey \\ ${ }^{5}$ Institute of Space Sciences, Magurele, Bucharest, Romania \\ ${ }^{6}$ Department of Mathematics, University of Salerno, Via Ponte don Melillo, Fisciano, 84084 Salerno, Italy \\ ${ }^{7}$ Electronic and Information Technology Department, Jiangmen Polytechnic, Jiangmen 529090, China
}

Correspondence should be addressed to Yang Zhao; zhaoyang19781023@gmail.com

Received 25 February 2014; Accepted 7 March 2014; Published 1 April 2014

Academic Editor: Hari M. Srivastava

Copyright (C) 2014 Long-Fei Wang et al. This is an open access article distributed under the Creative Commons Attribution License, which permits unrestricted use, distribution, and reproduction in any medium, provided the original work is properly cited.

We suggest a new model of the scale conservation equation in the mathematical theory of vehicular traffic flow on the fractal network based on the local fractional calculus.

\section{Introduction}

Continuum model was applied to describe the traffic flow with the continuous functions which was analogous to one of fluid dynamics and material models based on the conservation laws. The approach started with Lighthill, Whitham, and Richards's (LWR) works $[1,2]$. The LWR model was studied by many authors, for example, Daganzo [3], Zhang [4], Li [5], Gasser [6], Aw et al. [7], Bellomo and Coscia [8], and Bellomo et al. [9]. The LWR model of kinematic traffic waves derived from the conservation laws was [1-9]

$$
\frac{\partial^{\alpha}}{\partial t^{\alpha}} \rho+\frac{\partial^{\alpha}}{\partial x^{\alpha}} \phi=0
$$

where the quantity $u$ is the density of time $t$ and space $x$ and the quantity $\phi$ is the vehicle flux as a function of density $u$ and speed $v$ with $\phi=u v$, and its solution for the equation was discussed by using the finite difference method [10].

Starting with Mandelbrot [11] there were many reports to determine the fractal structure of nature in various fields of science and engineering. We recall that the geometric similarity of traffic networks was reported by many researchers, for example, Erramilli et al. [12], Lam and Wornell [13], Shang et al. [14], Li et al. [15], and Campari and Levi [16]. Recently, the local fractional calculus suggested in [17] was applied to deal with the nondifferentiable phenomena [1822]. For example, local fractional Navier-Stokes equations were suggested in [18] and the local fractional Helmholtz and diffusion equations were reported in [19]. The local fractional Maxwell's equations were proposed in [20]. Local fractional nonhomogeneous heat equations were investigated in [21]. The heat transfer in silk cocoon hierarchy with local fractional derivative was proposed in [22]. When the physical quantity of density or speed (denoted in Figure 1 by $f(x, t)$ ) for vehicular traffic flow on the fractal network is a nondifferentiable function with time and space defined on Cantor sets, the classical conservation law is no valid.

In order to overcome the above drawback, in this paper we discuss the fractal dynamical model of vehicular traffic flow within the local fractional conservation laws. The outline of the paper is as follows. In Section 2, we recall the local 


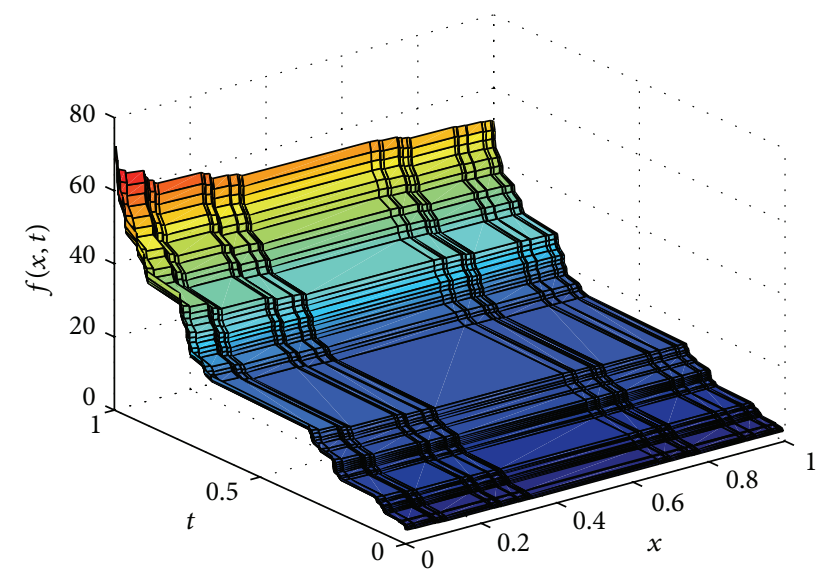

Figure 1: The nondifferentiable physical quantity $f(x, t)$ defined in Cantor sets with time $t$ and space $x$.

fractional conservation laws. In Section 4, a mathematical model of vehicular traffic flow with fractal network is suggested. In Section 5, the nonhomogeneous partial differential equations for the vehicular traffic flow with fractal network are discussed. The conclusions are shown in Section 5.

\section{Local Fractional Conservation Laws}

In this section, we introduce the local fractional conservation laws based on the local fractional calculus. We start with the conception of the local fractional vector integrals used in the paper.

Definition 1. The local fractional surface integral is defined as given below [17-21]:

$$
\iint u\left(r_{P}\right) d \mathbf{S}^{(\beta)}=\lim _{N \rightarrow \infty} \sum_{P=1}^{N} u\left(r_{P}\right) \mathbf{n}_{P} \Delta S_{P}^{(\beta)},
$$

where the quantity $\Delta S_{P}^{(\beta)}$ is the elements of fractal surface, $N$ is the elements of area with a unit normal local fractional vector $\mathbf{n}_{P}$, and $\Delta S_{P}^{(\beta)} \rightarrow 0$ as $N \rightarrow \infty$.

Definition 2. The local fractional volume integral of the function $\mathbf{u}$ is defined as [17-21]

$$
\iiint \mathbf{u}\left(r_{P}\right) d V^{(\gamma)}=\lim _{N \rightarrow \infty} \sum_{P=1}^{N} \mathbf{u}\left(r_{P}\right) \Delta V_{P}^{(\gamma)},
$$

where the quantity $\Delta V_{P}^{(\gamma)}$ is the elements of fractal volume and $\Delta V_{P}^{(\gamma)} \rightarrow 0$ as $N \rightarrow \infty$.

Definition 3. The local fractional gradient of the scale function $\phi$ has the form $[17,20]$

$$
\nabla^{\alpha} \phi=\lim _{d V^{(\gamma)} \rightarrow 0}\left(\frac{1}{d V^{(\gamma)}} \oiint_{S^{(\beta)}} \phi d \mathbf{S}^{(\beta)}\right)
$$

where the quantity $\nabla^{\alpha}$ is the local fractional Laplace operator [17], $\gamma=3 \alpha$, and $\beta=2 \alpha$.
The local fractional Gauss theorem of the fractal vector field states that [17-21]

$$
\iiint_{V^{(\gamma)}} \nabla^{\alpha} \cdot \mathbf{u} d V^{(\gamma)}=\oiint_{S^{(\beta)}} \mathbf{u} \cdot d \mathbf{S}^{(\beta)} .
$$

The integral form of local fractional conservation laws in fractal flow was suggested as $[17,21]$

$$
\frac{d^{\alpha}}{d t^{\alpha}} \iiint_{V^{(\gamma)}} \rho(r, t) d V^{(\gamma)}=-\oiint_{S^{(\beta)}} \sigma(r, t) \cdot d \mathbf{S}^{(\beta)}
$$

where the quantity $\rho(r, t)$ is the density of the material, the equality $\sigma(r, t)$ is the fractal flux vector, and $\sigma(r, t)=\rho(r, t) v$.

By using (5), (6) can be rewritten as

$$
\iiint_{V^{(\gamma)}}\left\{\frac{\partial^{\alpha} \rho(r, t)}{\partial t^{\alpha}}+\nabla^{\alpha} \cdot \sigma(r, t)\right\} d V^{(\gamma)}=0 .
$$

For any arbitrary volume, from (6) we obtain the local fractional differential form of the local fractional conservation laws as

$$
\frac{\partial^{\alpha} \rho(r, t)}{\partial t^{\alpha}}+\nabla^{\alpha} \cdot \sigma(r, t)=0
$$

In view of (8), the local fractional conservation law in the direction $x$ reads as follows:

$$
\frac{\partial^{\alpha} \rho(x, t)}{\partial t^{\alpha}}+\frac{\partial^{\alpha} \sigma(x, t)}{\partial x^{\alpha}}=0,
$$

where the fractal flux denotes $\sigma(x, t)=\rho(x, t) v(x, t)$ and the local fractional partial derivative of $f(x, t)$ of order $\alpha$ is defined as [17]

$$
\frac{\partial^{\alpha} f\left(x_{0}, t\right)}{\partial x^{\alpha}}=\frac{\Delta^{\alpha}\left(f(x, t)-f\left(x_{0}, t\right)\right)}{\left(x-x_{0}\right)^{\alpha}},
$$

where

$$
\Delta^{\alpha}\left(f(x, t)-f\left(x_{0}, t\right)\right) \cong \Gamma(1+\alpha)\left[f(x, t)-f\left(x_{0}, t\right)\right] .
$$

We recall the local fractional conservation laws, which had been successfully applied to deal with elasticity [17], fluid mechanics [18], diffusion [19], electromagnetic [20] and heat [21] flows, and so on.

\section{Fractal Dynamical Model of Vehicular Traffic Flow with Network}

In this section, we study the fractal dynamical model of vehicular traffic flow with network. In order to derive it, we consider that the number of vehicles on the fractal homogeneous road without sources and sinks is always conserved and that fractal flow is a product of the density and speed, which is a differentiable function. We now start with the derivation of the local fractional conservative law shown in Figure 2. 


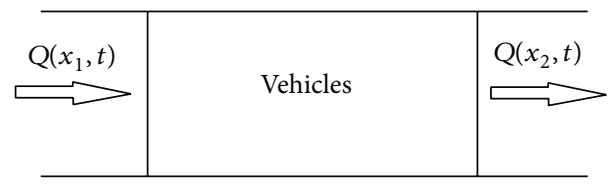

Figure 2: A segment of road is from $x_{1}$ to $x_{2}$. The fractal flow in at the position $x_{1}$ is $Q\left(x_{1}, t\right)$ and the fractal flow out at the position $x_{2}$ is $Q\left(x_{2}, t\right)$.

The local fractional integral of $f(x)$ of order $\alpha$ in the interval $[a, b]$ is given by $[17]$

$$
\begin{aligned}
{ }_{a_{b}}{ }^{(\alpha)} f(x) & =\frac{1}{\Gamma(1+\alpha)} \int_{a}^{b} f(t)(d t)^{\alpha} \\
& =\frac{1}{\Gamma(1+\alpha)} \lim _{\Delta t \rightarrow 0} \sum_{j=0}^{j=N-1} f\left(t_{j}\right)\left(\Delta t_{j}\right)^{\alpha},
\end{aligned}
$$

where the partitions of the interval $[a, b]$ are denoted as $\left(t_{j}, t_{j+1}\right), j=0, \ldots, N-1, t_{0}=a$, and $t_{N}=b$ with $\Delta t_{j}=$ $t_{j+1}-t_{j}$ and $\Delta t=\max \left\{\Delta t_{0}, \Delta t_{1}, \Delta t_{j}, \ldots\right\}$. The number of the vehicular traffics in the segment $x_{1}<x<x_{2}$ at time $t$ is

$$
\frac{1}{\Gamma(1+\alpha)} \int_{x_{1}}^{x_{2}} \varphi(x, t)(d x)^{\alpha}=N\left(x_{2}, t\right)-N\left(x_{1}, t\right),
$$

which leads to

$$
\frac{1}{\Gamma(1+\alpha)} \int_{x_{1}}^{x_{2}} \frac{\partial^{\alpha} \varphi(x, t)}{\partial t^{\alpha}}(d x)^{\alpha}=Q\left(x_{2}, t\right)-Q\left(x_{1}, t\right),
$$

where the density of traffic flow is $\varphi(x, t)$ and the quantities of traffic flux in fixed time $t$ are

$$
\begin{gathered}
\frac{\partial^{\alpha}}{\partial t^{\alpha}} N\left(x_{1}, t\right)=Q\left(x_{1}, t\right), \\
\frac{\partial^{\alpha}}{\partial t^{\alpha}} N\left(x_{2}, t\right)=Q\left(x_{2}, t\right) .
\end{gathered}
$$

Let us consider

$$
\frac{1}{\Gamma(1+\alpha)} \int_{x_{1}}^{x_{2}} \frac{\partial^{\alpha} Q(x, t)}{\partial x^{\alpha}}(d x)^{\alpha}=Q\left(x_{2}, t\right)-Q\left(x_{1}, t\right) .
$$

In view of (14) and (16), we have

$$
\frac{1}{\Gamma(1+\alpha)} \int_{x_{1}}^{x_{2}}\left\{\frac{\partial^{\alpha} \varphi(x, t)}{\partial t^{\alpha}}+\frac{\partial^{\alpha} Q(x, t)}{\partial x^{\alpha}}\right\}(d x)^{\alpha}=0 ;
$$

therefore,

$$
\frac{\partial^{\alpha} \varphi(x, t)}{\partial t^{\alpha}}+\frac{\partial^{\alpha} Q(x, t)}{\partial x^{\alpha}}=0,
$$

where $t>0$ and $x_{1}, x_{2} \in R$ are arbitrary. Equation (18) represents the Lighthill-Whitham-Richards model of fractal traffic flow with local fractional derivative. We notice that (18) is in agreement with (9).
For the traffic flow $Q(x, t)=\varphi(x, t) v(x, t),(18)$ can be written as

$$
\frac{\partial^{\alpha} \varphi}{\partial t^{\alpha}}+\frac{\partial^{\alpha}}{\partial x^{\alpha}}(\varphi v)=0
$$

with the initial value condition

$$
\varphi(x, 0)=\varphi_{0}(x) .
$$

We denote the traffic flow by $Q=Q(\varphi)$; thus,

$$
Q(\varphi)=\varphi v(\varphi) .
$$

With the help of (21) and by using (19) we conclude that

$$
\frac{\partial^{\alpha} \varphi}{\partial t^{\alpha}}+\eta(\varphi) \frac{\partial^{\alpha} \varphi}{\partial x^{\alpha}}=0
$$

where

$$
\eta(\varphi)=Q^{(1)}(\varphi) .
$$

If the velocity $v=v(\varphi)=\mu$ is constant, then from (22) and (23) we get the Cauchy problem of the linear LighthillWhitham-Richards model given by

$$
\frac{\partial^{\alpha} \varphi(x, t)}{\partial t^{\alpha}}+\mu \frac{\partial^{\alpha} \varphi(x, t)}{\partial x^{\alpha}}=0
$$

subject to the initial value condition

$$
\varphi(x, 0)=\varphi_{0}(x) .
$$

From (24), we easily obtain the Lighthill-WhithamRichards model on a finite length highway

$$
\frac{\partial^{\alpha} \varphi(x, t)}{\partial t^{\alpha}}+\mu \frac{\partial^{\alpha} \varphi(x, t)}{\partial x^{\alpha}}=0
$$

with the initial and boundary conditions

$$
\begin{gathered}
\varphi(x, 0)=\varphi_{0}(x) . \\
\varphi(0, t)=\omega(t) .
\end{gathered}
$$

This equation is the liner Lighthill-Whitham-Richards model of fractal traffic flow with local fractional derivative.

Let us consider a linear velocity given as

$$
v=v(\varphi)=\xi+\eta \varphi
$$

then, the expression of the traffic flow becomes

$$
Q(x, t)=\xi \varphi(x, t)+\eta \varphi^{2}(x, t),
$$

where $\xi$ is the fractal unimpeded traffic speed and $\eta$ represents the maximum density.

From (19) and (29) we obtain the nonlinear local fractional partial differential equation

$$
\frac{\partial^{\alpha} \varphi(x, t)}{\partial t^{\alpha}}+\xi \frac{\partial^{\alpha} \varphi(x, t)}{\partial x^{\alpha}}+2 \eta \varphi(x, t) \frac{\partial^{\alpha} \varphi(x, t)}{\partial x^{\alpha}}=0,
$$


where $\varphi(x, t)$ is density of fractal traffic flow. This equation is the nonlinear Lighthill-Whitham-Richards model of fractal traffic flow with local fractional derivative.

From (30) we derive Cauchy problem of the nonlinear Lighthill-Whitham-Richards model of fractal traffic flow

$$
\frac{\partial^{\alpha} \varphi(x, t)}{\partial t^{\alpha}}+\xi \frac{\partial^{\alpha} \varphi(x, t)}{\partial x^{\alpha}}+2 \eta \varphi(x, t) \frac{\partial^{\alpha} \varphi(x, t)}{\partial x^{\alpha}}=0,
$$

such that

$$
\varphi(x, 0)=\varphi_{0}(x) .
$$

From (30), the initial-boundary problem for nonlinear Lighthill-Whitham-Richards model of fractal traffic flow is suggested as

$$
\begin{aligned}
\frac{\partial^{\alpha} \varphi(x, t)}{\partial t^{\alpha}}+\xi \frac{\partial^{\alpha} \varphi(x, t)}{\partial x^{\alpha}} & +2 \eta \varphi(x, t) \frac{\partial^{\alpha} \varphi(x, t)}{\partial x^{\alpha}}=0, \\
\varphi(x, 0) & =\varphi_{0}(x), \\
\varphi(0, t) & =\omega(t),
\end{aligned}
$$

provided that [17]

$$
\begin{gathered}
\left|\varphi_{0}(x)-\varphi_{0}\left(x_{0}\right)\right|<\varepsilon^{\alpha}, \\
\left|\omega(t)-\omega\left(t_{0}\right)\right|<\kappa^{\alpha},
\end{gathered}
$$

with $\left|x-x_{0}\right|<\delta,\left|t-t_{0}\right|<\tau$ for $\varepsilon, \kappa, \delta, \tau>0,0<\alpha<1$.

\section{Discussions}

In this section, we investigate the nonhomogeneous partial differential equations for the vehicular traffic flow with fractal network.

From (24) and (25), the Cauchy problem of the nonhomogeneous partial differential equation of Lighthill-WhithamRichards model with nondifferentiable source term $\psi(x, t)$ is given by

$$
\frac{\partial^{\alpha} \varphi(x, t)}{\partial t^{\alpha}}+\mu \frac{\partial^{\alpha} \varphi(x, t)}{\partial x^{\alpha}}=\psi(x, t),
$$

subject to the initial value condition

$$
\varphi(x, 0)=\varphi_{0}(x) .
$$

In view of (26) and (27), the initial and boundary problems for the nonhomogeneous partial differential equation of Lighthill-Whitham-Richards model with nondifferentiable source term $\psi(x, t)$ on a finite length highway become

$$
\frac{\partial^{\alpha} \varphi(x, t)}{\partial t^{\alpha}}+\mu \frac{\partial^{\alpha} \varphi(x, t)}{\partial x^{\alpha}}=\psi(x, t)
$$

with the initial and boundary conditions

$$
\begin{gathered}
\varphi(x, 0)=\varphi_{0}(x), \\
\varphi(0, t)=\omega(t) .
\end{gathered}
$$

Considering (31) and (32), the Cauchy problem of the nonhomogeneous nonlinear partial differential equation of Lighthill-Whitham-Richards model with nondifferentiable source term $\psi(x, t)$ becomes

$$
\frac{\partial^{\alpha} \varphi(x, t)}{\partial t^{\alpha}}+\xi \frac{\partial^{\alpha} \varphi(x, t)}{\partial x^{\alpha}}+2 \eta \varphi(x, t) \frac{\partial^{\alpha} \varphi(x, t)}{\partial x^{\alpha}}=\psi(x, t),
$$

with the initial value condition

$$
\varphi(x, 0)=\varphi_{0}(x)
$$

By taking into account (33), the initial-boundary problem for nonhomogeneous nonlinear partial differential equation of Lighthill-Whitham-Richards model with nondifferentiable source term $\psi(x, t)$ can be written as

$$
\begin{gathered}
\frac{\partial^{\alpha} \varphi(x, t)}{\partial t^{\alpha}}+\xi \frac{\partial^{\alpha} \varphi(x, t)}{\partial x^{\alpha}}+2 \eta \varphi(x, t) \frac{\partial^{\alpha} \varphi(x, t)}{\partial x^{\alpha}}=\psi(x, t), \\
\varphi(x, 0)=\varphi_{0}(x) \\
\varphi(0, t)=\omega(t),
\end{gathered}
$$

subjected to

$$
\begin{gathered}
\left|\varphi_{0}(x)-\varphi_{0}\left(x_{0}\right)\right|<\varepsilon^{\alpha}, \\
\left|\omega(t)-\omega\left(t_{0}\right)\right|<\kappa^{\alpha},
\end{gathered}
$$

with $\left|x-x_{0}\right|<\delta,\left|t-t_{0}\right|<\tau$ for $\mathcal{E}, \kappa, \delta, \tau>0,0<\alpha<1$.

\section{Conclusions}

In this work, the fractal dynamical models of vehicular traffic flow within the local fractional conservation laws, where the density and speed of fractal traffic flow are nondifferentiable functions, are investigated. Besides, the linear and nonlinear partial differential equations for Lighthill-Whitham-Richards models of the vehicular traffic flows with fractal networks are obtained. The classical results are special case of the ones with nondifferentiable conditions when the fractal dimension $\alpha$ is equal to 1 .

\section{Conflict of Interests}

The authors declare that there is no conflict of interests regarding the publication of this paper.

\section{Acknowledgments}

This work was supported by the Fundamental Research Funds for the Central Universities under Grant no. CHD2011JC191. It was also supported by the National Natural Science Foundation of China under Grant no. 51208054. 


\section{References}

[1] M. J. Lighthill and G. B. Whitham, "On kinematic waves-II. A theory of traffic flow on long crowded roads," Proceedings of the Royal Society of London A: Mathematical, Physical and Engineering Sciences, vol. 229, no. 1178, pp. 317-345, 1955.

[2] P. I. Richards, "Shock waves on the highway," Operations Research, vol. 4, no. 1, pp. 42-51, 1956.

[3] C. F. Daganzo, "A continuum theory of traffic dynamics for freeways with special lanes," Transportation Research B: Methodological, vol. 31, no. 2, pp. 83-102, 1997.

[4] H. M. Zhang, "New perspectives on continuum traffic flow models," Networks and Spatial Economics, vol. 1, no. 1-2, pp. 933, 2001.

[5] T. Li, " $L^{1}$ stability of conservation laws for a traffic flow model," Electronic Journal of Differential Equations, vol. 2001, no. 14, pp. $1-18,2001$.

[6] I. Gasser, "On non-entropy solutions of scalar conservation laws for traffic flow," Journal of Applied Mathematics and Mechanics, vol. 83, no. 2, pp. 137-143, 2003.

[7] A. Aw, A. Klar, T. Materne, and M. Rascle, "Derivation of continuum traffic flow models from microscopic follow-theleader models," SIAM Journal on Applied Mathematics, vol. 63, no. 1, pp. 259-278, 2002.

[8] N. Bellomo and V. Coscia, "First order models and closure of the mass conservation equation in the mathematical theory of vehicular traffic flow," Comptes Rendus Mecanique, vol. 333, no. 11, pp. 843-851, 2005.

[9] N. Bellomo, M. Delitala, and V. Coscia, "On the mathematical theory of vehicular traffic flow-I: fluid dynamic and kinetic modelling," Mathematical Models and Methods in Applied Sciences, vol. 12, no. 12, pp. 1801-1843, 2002.

[10] C. F. Daganzo, "A finite difference approximation of the kinematic wave model of traffic flow," Transportation Research B: Methodological, vol. 29, no. 4, pp. 261-276, 1995.

[11] B. B. Mandelbrot, The Fractal Geometry of Nature, W. H. Freeman, San Francisco, Calif, USA, 1982.

[12] A. Erramilli, W. Willinger, and P. Pruthi, "Fractal traffic flows in high-speed communications networks," Fractals, vol. 2, no. 3, pp. 409-412, 1994.

[13] W. M. Lam and G. W. Wornell, "Multiscale analysis and control of networks with fractal traffic," Applied and Computational Harmonic Analysis, vol. 11, no. 1, pp. 124-146, 2001.

[14] P. Shang, M. Wan, and S. Kama, "Fractal nature of highway traffic data," Computers \& Mathematics with Applications, vol. 54, no. 1, pp. 107-116, 2007.

[15] M. Li, W. Zhao, and C. Cattani, "Delay bound: fractal traffic passes through network servers," Mathematical Problems in Engineering, vol. 2013, Article ID 157636, 15 pages, 2013.

[16] E. G. Campari and G. Levi, "Self-similarity in highway traffic," The European Physical Journal B: Condensed Matter and Complex Systems, vol. 25, no. 2, pp. 245-251, 2002.

[17] X.-J. Yang, Advanced Local Fractional Calculus and Its Applications, World Science, New York, NY, USA, 2012.

[18] X.-J. Yang, D. Baleanu, and J. A. T. Machado, "Systems of NavierStokes equations on Cantor sets," Mathematical Problems in Engineering, vol. 2013, Article ID 769724, 8 pages, 2013.

[19] Y.-J. Hao, H. M. Srivastava, H. Jafari, and X.-J. Yang, "Helmholtz and diffusion equations associated with local fractional derivative operators involving the Cantorian and Cantor-type cylindrical coordinates," Advances in Mathematical Physics, vol. 2013, Article ID 754248, 5 pages, 2013.
[20] Y. Zhao, D. Baleanu, C. Cattani, D.-F. Cheng, and X.-J. Yang, "Maxwell's equations on Cantor sets: a local fractional approach," Advances in High Energy Physics, vol. 2013, Article ID 686371, 6 pages, 2013.

[21] A.-M. Yang, C. Cattani, C. Zhang, G.-N. Xie, and X.-J. Yang, "Local fractional Fourier series solutions for non-homogeneous heat equations arising in fractal heat flow with local fractional derivative," Advances in Mechanical Engineering, vol. 2014, Article ID 514639, 5 pages, 2014.

[22] J.-H. He and F.-J. Liu, "Local fractional variational iteration method for fractal heat transfer in silk cocoon hierarchy," Nonlinear Science Letters A, vol. 4, no. 1, pp. 15-20, 2013. 


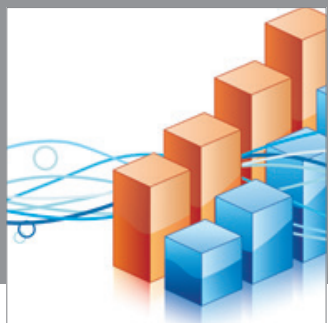

Advances in

Operations Research

mansans

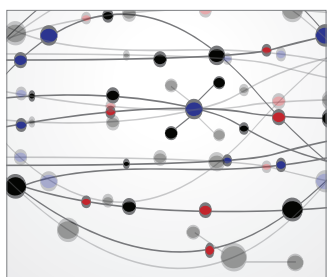

The Scientific World Journal
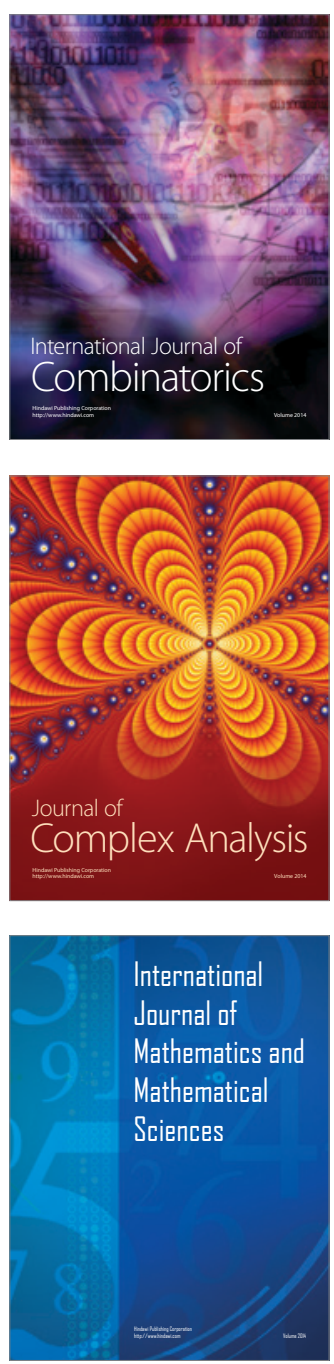
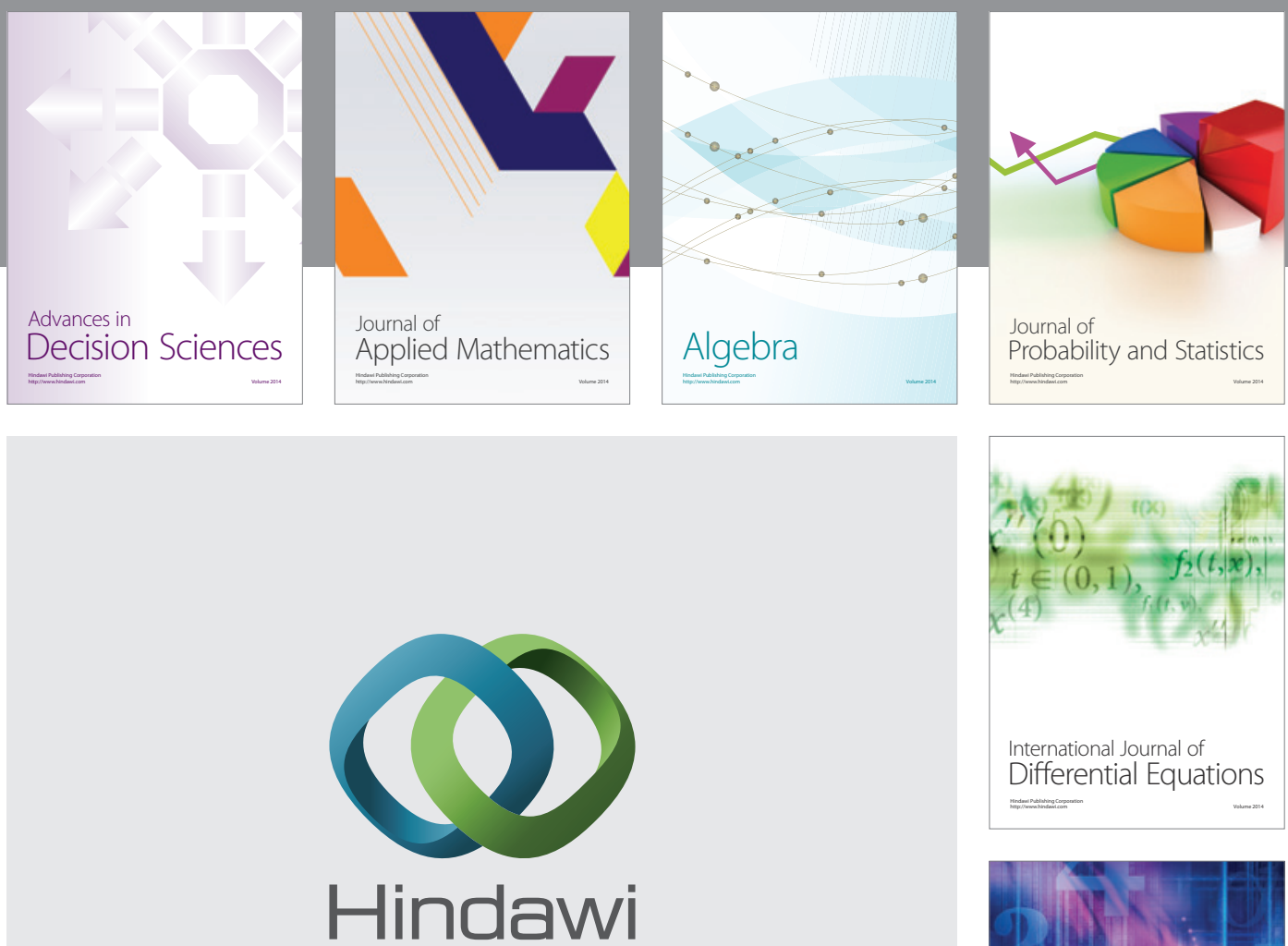

Submit your manuscripts at http://www.hindawi.com
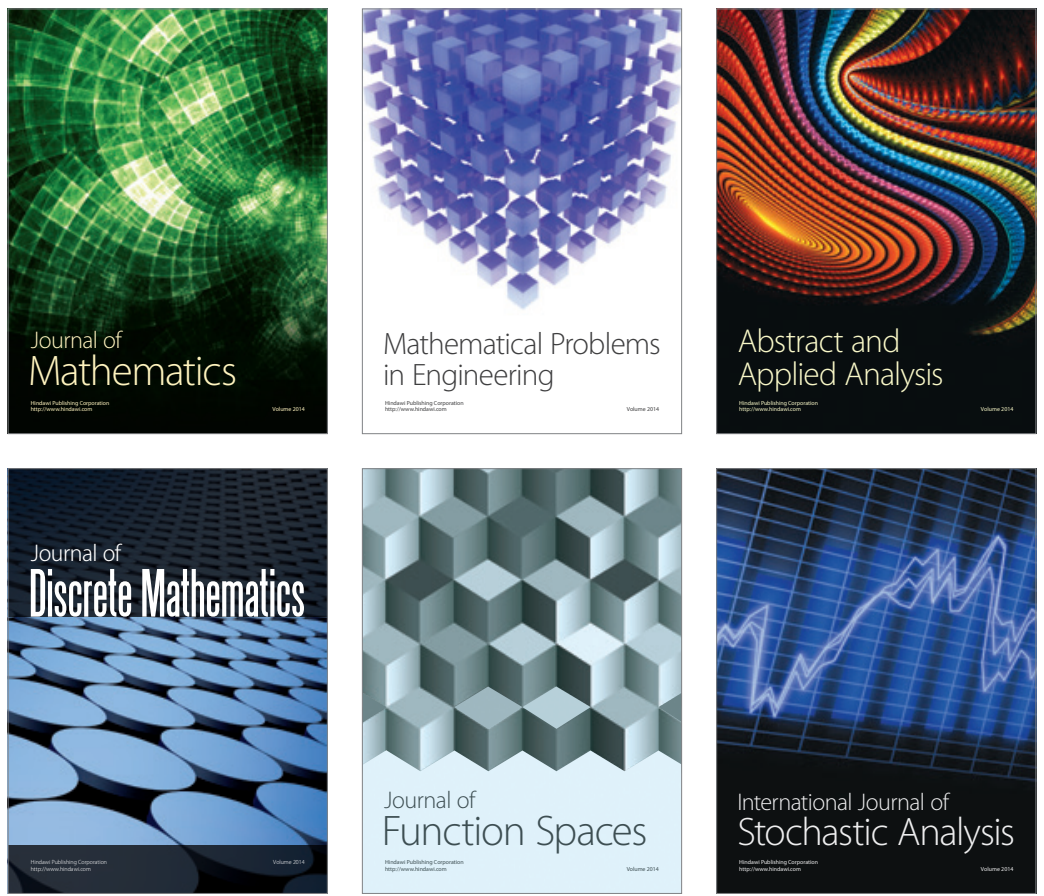

Journal of

Function Spaces

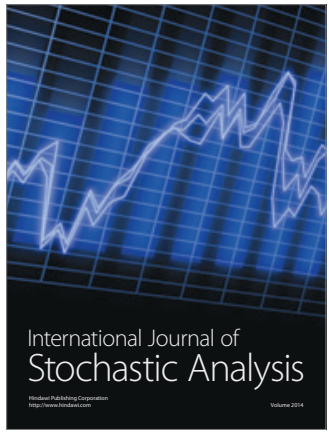

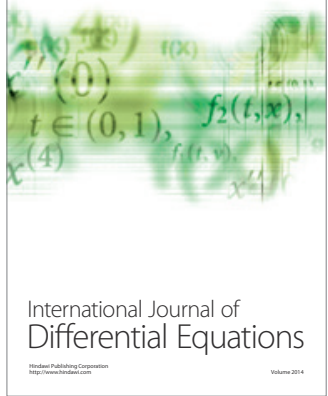
